\title{
Impact of dexmedetomidine on amino acid contents and the cerebral ultrastructure of rats with cerebral ischemia-reperfusion injury ${ }^{1}$
}

Sheng Lin', Guangli Zhou', Wei Shao', Zhijian Fu"

'MD, Department of Anesthesiology, Yantaishan Hospital, China. Conception and design of the study, manuscript writing, final approved.

"MD, Department of Pain, Provincial Hospital, Shandong University, China. Analysis of data, final approved.

\section{Abstract}

Purpose: To investigate the effects of dexmedetomidine (DEX) on amino acid contents and the cerebral ultrastructure of rats with cerebral ischemia-reperfusion injury (I/R).

Methods: Thirty-six, male, Wistar rats were randomly divided into three groups: the sham operation group (group C), the ischemia-reperfusion group (group I/R), and the DEX group (group D). The middle cerebral artery occlusion model was prepared by the modified Longa method. The time of ischemia was $180 \mathrm{~min}$, and $120 \mathrm{~min}$ after reperfusion, the amount of glutamate (Glu), and $\gamma$-aminobutyric acid (GABA) in the brain were measured, and the ultrastructure-level changes in the cerebral cortex were examined using electron microscopy.

Results: Compared to group C, Glu contents in group D, and I/R significantly increased. Compared to group I/R, Glu contents in group D significantly decreased. Compared to group C, GABA contents in group D, and I/R significantly increased, and those in group D significantly increased, as compared to group I/R. The cerebral ultrastructure was normal in group C. Vacuolar degeneration in the plastiosome and nervous processes, was more critical than in group D. Vascular endothelial cells (VEC) were damaged. On the contrary, these changes in group D significantly improved.

Conclusion: Dexmedetomidine is capable of decreasing glutamergic content, and increasing GABAergic content, in order to decrease the injury of the cerebral ultrastructure, following cerebral ischemia-reperfusion injury.

Key words: Medetomidine. Amino Acids. Brain Ischemia. Reperfusion. Rats. 


\section{Introduction}

The central nervous system (CNS) has a large number of amino acids, which play important roles in the conduction process of nerve functions, by working as neurotransmitters. Based on the excitatory or inhibitory effects of postsynaptic neurons, amino acids could be divided into two types: excitatory amino acids (EAAs), and inhibitory amino acids (IAA). The maintenance of excitability, or the lack thereof in the CNS, depends on the relative proportions of these two types of neurotransmitters. EAAs mainly refer to glutamate (Glu), and aspartate (Asp), and IAA mainly refer to $\gamma$-aminobutyric acid (GABA), and glycine (Gly).

EAAs, especially Glu, play important roles in cerebral ischemia-reperfusion injury (I/R) injury. Gluis an important neurotransmitter responsible to mediate rapid synaptic responses within the CNS, and only a small amount of Glu exists in the synaptic cleft of brain tissues, under normal circumstances. Its metabolic balance is mainly achieved by a normal release of Glu from the presynaptic membrane, and the subsequent reuptake by neurons, and glial cells. Imbalances in this process, of Glu release and uptake, could aggravate cerebral I/R injury $^{1,2}$. In cerebral ischemia, the presynaptic membrane would be in a depolarized state, leading to a massive release of Glu. Meanwhile, due to a lack of adenosine triphosphate (ATP), the neuron-glial cell signaling transduction would be disrupted, followed by a reuptake disorder of glial cells, the accumulation of Glu among cells, and an excessive activation of Glu receptors on the postsynaptic membrane, of which the most important is the $\mathrm{N}$-methylD-aspartate (NMDA) receptor. An excessive activation of the NMDA receptors causes cell death, primarily through mechanisms such as $\mathrm{Ca}^{2+}$-overload associated secondary mitochondrial dysfunctions, an increasing amount of reactive oxygen species, abnormal transcription, and protease and endonuclease activation $^{3}$. Previous experiments, conducted in vivo or at the cellular level have also shown that in addition to cerebral ischemia, Glu is also responsible for the activation of Glu receptors on the postsynaptic membrane, generation of excitotoxicity, and causing neuronal damage in the pathological processes of trauma, and CNS chronic degenerative diseases ${ }^{4}$.

Dexmedetomidine (DEX) is a highly selective $\alpha 2$-adrenoceptor agonist5. A study found that DEX has neuroprotective effects, as it could improve oxygenation following cerebral $I / R$, reduce the cerebral infarction area, and reduce the apoptosis of neuronal cells ${ }^{6-8}$. Some in vitro studies have also found that DEX could inhibit astrocytes and thus increase the oxidative metabolism of the astrocytes towards Glu, thus preventing Glu to be metabolized to glutamine ${ }^{9,10}$. However, the direct effects of DEX on brain Glu were not reported. In order to investigate this, we hypothesized that DEX could potentially reduce the content of Glu in rats with cerebral I/R injury, thus playing neuroprotective effects.

\section{Methods}

This study was carried out in strict accordance with the recommendations in the Guide for the Care and Use of Laboratory Animals of the National Institutes of Health. The animal use protocol has been reviewed and approved by the Institutional Animal Care and Use Committee (IACUC) of Yantaishan Hospital.

Thirty-six, healthy, male, Wistar rats (clean-grade, body weight 250-300g, provided by Shandong Luye Pharmaceutical Co., Ltd., animal Certificate of Conformity: SYXK (Lu) 20090013) were selected one week before surgery; they had free access to water, and a standard diet. The laboratory was maintained 
at a constant temperature $\left(\sim 23^{\circ} \mathrm{C}\right)$. Rats were made to fast, with no prohibitions on drinking water, $12 \mathrm{~h}$ before the surgery; then, the animals were randomly divided into three groups: 1) the sham operation group (group C): we only exposed the external carotid artery surgically, not causing ischemia; 2) the I/R group: we intraperitoneally injected $1 \mathrm{~mL}$ of sterile saline, $30 \mathrm{~min}$ before the ischemia operation, followed by cerebral ischemia operation; 3) the DEX group (group D): we intraperitoneally injected DEX $100 \mu \mathrm{g} / \mathrm{kg}$ (Jiangsu Hengrui Medicine Co., Ltd.), $30 \mathrm{~min}$ before the ischemia operation, followed by cerebral ischemia operation. The ischemia time was the same as in the I/R group, and $120 \mathrm{~min}$ after reperfusion, all rats were decapitated, and their hippocampi were sampled for the content detection of Glu, and GABA.

\section{Model preparation}

The $I / R$ model was prepared in the left middle cerebral artery using the modified Longa method11, which could induce focal lesion in the MCA feeding area. Briefly, mice were subjected to anesthesia using $5.0 \%$ Isoflurane, and this was maintained by inhalation of $1.5 \%$ to $2 \%$ Isoflurane, driven by $100 \%$ oxygen flow. Mice were ventilated (110 breaths/min with volume $0.5 \mathrm{~mL}$ ), and body temperature was regulated at $37^{\circ} \mathrm{C}$, by surface water heating. We cut the neck fur, prepared the skin, and performed the median incision, then, transversely incised the skin and carefully separated tissue layers to expose the sternocleidomastoid. Following this, we bluntly dissected the sternocleidomastoid, and sternohyoid, using surgical retractors. After exposing the left common carotid artery (CCA), we gently separated upwards along the common carotid artery until it was $1 \mathrm{~cm}$ away from the distal end of the left external carotid artery (ECA), then ligated the ECA branches, and ligated ECA at the CCA crotches.
This was followed by clamping the proximal end of CCA using artery clamps, penetrating a suture near the bottom of the ECA crotches, and then tying the knot without tightening it. Then, we prepared a small incision between the proximal end of the ECA ligation, and the CCA crotch, gently inserted the nylon suture prepared in advance through the incision, and gently tightened the knot. Following this, we loosened the artery clamp to restore the blood flow, sent the nylon suture along the ECA into the brain, until we felt slight resistance, at which point the nylon suture may have been inserted about 17 19 mm inside. At this point, the tip of the nylon suture had already reached the MCA end; hence, we gently tightened the suture head to completely block the blood supply of MCA. When the iridal color of the rat became lighter, and Horner's syndrome (characterized by ptosis, miosis, "upside-down ptosis" [or elevation of the lower lid], and facial anhydrosis) occurred, we considered that the model preparation was successful. Then, we sutured the incision layer by layer, and left approximately $1 \mathrm{~cm}$ of the nylon suture out of the incision. The whole process was completed within $10 \mathrm{~min}$. After the $180 \mathrm{~min}$ ischemia, we re-injected $10 \%$ chloral hydrate to anesthetize the rat, lightly withdrew the reserved nylon suture until we felt resistance, at which point we considered that its head had returned to the CCA crotch. In this case, the brain was restored with blood supply, and we achieved reperfusion. Finally, we cut the excessive suture to complete preparing the MCAO model. Group C was only anesthetized with $10 \%$ chloral hydrate, and in this case, we separated the left ECA until $1 \mathrm{~cm}$ away from the distal end, while not performing vessel ligation, and suture introduction. The remaining part of the operation was the same as the I/R group. The DEX group was premedicated according to experimental design, and the remaining operations were the same as the I/R group. We maintained the rats' body 
temperatures at $37^{\circ} \mathrm{C}$, postoperatively, and the animals had free access to food and water. We also observed their vital signs, and maintained airway patency.

\section{Specimen collection}

After $120 \mathrm{~min} \mathrm{I} / \mathrm{R}$, rats was reinjected with $10 \%$ chloral hydrate $(300 \mathrm{mg} /$ $\mathrm{kg}$ ), intraperitoneally, for anesthesia, and we quickly cut the chest, exposed the heart, cut the left ventricle, and cannulated into the ascending aorta level. After quickly injecting $200 \mathrm{~mL}$ physiological saline for flushing, we fixed samples with $4 \%$ paraformaldehyde, and cut the head to sample the brain. Then, fixed the brain in $4 \%$ paraformaldehyde for $24 \mathrm{~h}$, followed by conventional alcohol dehydration. Following this, we soaked the brain tissues in a paraffin box; after full fixation, we placed the embedding instrument on ice, adequately cooled the brain tissues, and performed continuous brain coronal frozen slicing, with the thickness set as $5 \mu \mathrm{m}$. Thereafter, we stored the samples at $4^{\circ} \mathrm{C}$ for future use.

\section{Observation of cell ultrastructure}

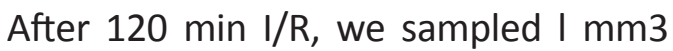
brain tissues at the junction of the striatum, and the cortex (the penumbra region), from the fresh rat brain coronal slices. Then, we stored samples in $2.5 \%$ glutaraldehyde at $4^{\circ} \mathrm{C}$, rinsed with phosphate buffer $15 \mathrm{~min} \times 3$ times, fixed with $0.1 \%$ osmium tetroxide for $1 \mathrm{~h}$, and dehydrated with $90 \%$, followed by $95 \%$, and finally $100 \%$ ethanol. This dehydration process was repeated with acetone, going from $90 \%$, to $95 \%$, and finally $100 \%$. Samples were further impregnated with epoxy resin, embedded, and fixed. We prepared $5 \mathrm{~nm}$ ultrathin sections with LKB ultramicrotome (Hubei Huida Instrument Co., Ltd. Wuhan, China), and observed ultrastructural changes using a staining transmission electron microscope (Hitachi, Japan).

\section{Detection of Glu and GABA}

We centrifuged the prepared brain tissues in a low-temperature, high-speed (4? ? $12000 \mathrm{r} / \mathrm{min}$ ) centrifuge for $10 \mathrm{~min}$ at low temperature. Then, we diluted the supernatant of the tissue homogenate with saline, detected and calculated the contents of Glu and GABA in strict accordance with the kit instructions, using a spectrophotometer (Shanghai Optical Instrument Factory; Glu and GABA Kit: Nanjing Jiancheng Bioengineering Institute).

\section{Statistical analysis}

All data were processed using SPSS (version 13.0) software; the measurement data were expressed as mean \pm standard deviation ( $\pm s)$. We used ANOVA for intra- and inter-group comparisons, at different time points, and the $q$ test for pairwise comparison among the averages, with $\mathrm{P}<0.05$ considered as statistically significant.

\section{- Results}

\section{Comparison of Glu and GABA content}

Compared to group C, the Glu contents in group $D$, and $I / R$ significantly increased $(P<0.05)$, and those in group $D$ were significantly lower than in group I/R $(P<0.05)$. Compared to group $C$, the GABA contents in group $D$, and $I / R$ were significantly reduced, and those in group D were significantly higher, compared to group I/R (Table 1).

Table 1 - Comparisons of Glu and GA B A content among the groups ( $n=12, \mu \mathrm{mol} /$ gprot).

\begin{tabular}{lcc}
\hline Group & Glu & GABA \\
\hline C & $176.6 \pm 23.4$ & $467.4 \pm 33.5$ \\
I/R & $434.5 \pm 46.4^{*}$ & $166.6 \pm 12.2 *$ \\
D & $367.6 \pm 34.2 * \#$ & $258.4 \pm 16.2 * \#$ \\
\hline
\end{tabular}

*Compared with group C, $\mathrm{P}<0.05$, \# compared with group $\mathrm{D}$, $\mathrm{P}<0.05$. 


\section{Ultrastructural changes}

The ultrastructure of the brain tissues in group $C$ was normal (Figure 1A). The neurons, nuclei, and nuclear membrane were complete, without edema around the nuclei. Peri-nuclear gaps were not widened, the nucleoli were obvious, and chromatin was distributed evenly. No vacuolar degeneration was seen in the mitochondria. The cytoplasm of the neurons, oligodendrocytes, and microglia in the cerebral cortex of the samples in the I/R group exhibited edema, and membrane structures were not complete, apart from being swollen, and deformed.Thecytoplasmexhibitedvacuolization, and the nucleoli had shrunk and dissolved, along with peri-nuclear regions exhibiting obvious edema, and widened perinuclear gaps. In addition, majority of the cell mitochondria exhibited vacuolar degeneration, the vascular endothelial cells were damaged, congestion was seen in capillaries, and the endothelial cells were damaged with visible congestion (Figure 1B). The neurons, oligodendrocytes, and microglia in group $D$ were intact, and the membrane structures were complete. Few mitochondria exhibited vacuolar degeneration; the gaps between the blood vessels and nervous processes (V-R gap) were slightly changed, the continuity of the vascular endothelial cells was complete, and no congestion was seen in the capillaries (Figure 1C).
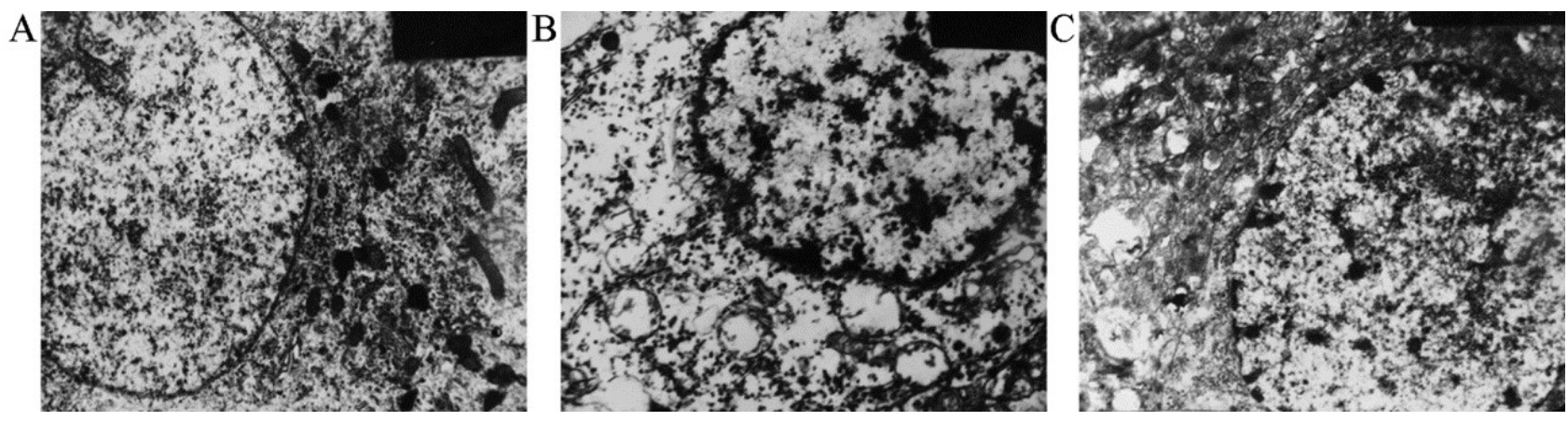

Figure 1 - A: Normal neurons. B: Neurons in the I/R group. C: The lesions in group D were significantly reduced (x7200).

\section{- Discussion}

There are many amino acids acting as neurotransmitters inside the CNS, and they are involved in a variety of nerve information transmissions among the neurons, and the maintenance of the intra- and intercellular distribution of water and ions. When the brain functions are normal, EAA and IAA are in a relatively balanced state. Of these, Glu plays a major role in cerebral I/R injury: in cerebral I/R injury, large amounts of Glu, usually stored in the presynaptic endings are released, causing a sharp increase in the Glu content within the extracellular fluid of the brain cells ${ }^{12}$. The normal brain cell membranes have a large number of highly affiliative excitatory amino acid transporters (EAATs), whose function is to transport EAA from the synaptic cleft into cells, thereby maintaining the amino acid balance within brain tissues. When in cerebral $I / R$ injury, the ion gradient inside and outside the cell membrane is reduced; therefore, the intake capacity of EAAs by EAATs reduces, resulting in EAAs reuptake disorder. The substantially increased Glu then acts on the EAA receptors on the postsynaptic membrane, thus causing an over-activation of the EAA receptors on the postsynaptic membrane, and the secondary neuronal injury ${ }^{13}$. GABA is a major inhibitory 
neurotransmitter in the brain, and could generate presynaptic or postsynaptic inhibition of neurons, when bound with its receptors ${ }^{14}$.

The levels of intra-cerebral EAA following acute cerebral I/R injury are closely related to the severity of the brain injury ${ }^{15,16}$. Studies have shown that Glu produces brain injury primarily through two ways: first, through the non-NMDA receptor, and second, through exciting the NMDA receptor, thus directly or indirectly starting the voltage-dependent Ca2+ channel, leading to an intracellular $\mathrm{Ca} 2+$ overload, and thus delayed neuronal degeneration, and necrosis ${ }^{17}$. The neurotoxicity of EAA is mainly expressed in the following ways: 1 , inducing lipid peroxidation towards the unsaturated fatty acids of the cell membrane, thus damaging the cell membrane ion channels and causing cell edema; 2 , causing a decrease or loss in the biological activities of a variety of enzymes ${ }^{18,19}$; and 3, reducing mitochondrial functions, disturbing energy metabolism, and causing apoptosis in some cells ${ }^{20,21}$.

Certain studies have shown ${ }^{22}$ that extracellular Glu concentrations are closely related to the severity, duration, organ sizes involved, and ischemic sites of cerebral ischemia, which usually gradually decline with the restoration of the blood flow; however, the concentration significantly increases, again, at approximately $120 \mathrm{~min}$ of the infusion recovery. Therefore, our study designed the brain sampling two hours later, after the blood flow is recovered.

DEX is a novel, highly selective a2adrenoceptor agonist, and its binding ratio with the $\alpha 2$ adrenergic receptor is $1620: 1$. It has inhibitory effects towards the sympathetic nervous system, such as analgesia, sedation, and antianxiety. Foreign scholars have applied DEX for sedation assistance in neurosurgical procedures, and it is known that its sedative effects can be easily reversed. Additionally, it also makes the patient tolerate the endotracheal tube ${ }^{23,24}$. DEX has a lighter suppression towards respiratory functions, and due to this characteristic, it is now used as a major adjunct for clinical anesthesia. Recent studies conducted on rabbits with subarachnoid hemorrhage found that DEX exhibits protective effects on hippocampal neurons ${ }^{25}$. We also found in our previous experiments that DEX could mitigate the apoptosis of rat brain cells. In this study, we found that DEX could reduce Glu content, while increasing the GABA content in brain tissues with cerebral I/R injury. DEX stimulates astrocyte $\alpha$ - 2 adrenergic receptors, which in turn raise astrocyte calcium concentrations. These elevated concentrations then stimulate glutaminase activity, and the ability of astrocytes to eliminate glutamine by oxidative metabolism, thereby reducing the availability of glutamine as a precursor of neurotoxic glutamate. Furthermore, DEX inhibits evoked glutamate release by stimulating $\alpha-2$ adrenergic receptors though a mechanism involving the suppression of Calcium voltagegated 2.2, and Calcium voltage-gated 2.1 channels, and mitogen-activated protein kinase activity. The results achieved using the electron microscope also show that DEX is capable of reducing damage in the brain tissues caused due to cerebral I/R injury, indicating that DEX might reduce apoptosis through the reduction of Glu content, and thus exert its neuroprotective effects.

\section{Conclusions}

Dexmedetomidine has the capability to reduce glutamergic content, and simultaneously increase GABAergic content, in order to reduce the damage to the cerebral ultrastructure, that occurs after cerebral ischemia.

\section{References}

1. Pu J, Niu X, Zhao J. Excitatory amino acid changes in the brains of rhesus monkeys following selective cerebral deep hypothermia and blood flow occlusion. Neural Regen Res. 2013 Jan 15;8(2):143-8. 
doi: 10.3969/j.issn.1673-5374.2013.02.006.

2. Sun X, Zhong J, Wang D, Xu J, Su H, An C, Zhu $\mathrm{H}$, Yan J. Increasing glutamate promotes ischemia-reperfusion-induced ventricular arrhythmias in rats in vivo. Pharmacology. 2014;93(1-2):4-9. doi: 10.1159/000356311.

3. Gibson CJ, Meyer RC, Hamm RJ. Traumatic brain injury and the effects of diazepam, diltiazem, and MK-801 on GABA-A receptor subunit expression in rat hippocampus. J Biomed Sci. 2010 May 18;17:38. doi: 10.1186/1423-0127-17-38.

4. Ruban A, Mohar B, Jona G, Teichberg VI. Blood glutamate scavenging as a novel neuroprotective treatment for paraoxon intoxication. J Cereb Blood Flow Metab. 2014 Feb;34(2):221-7. doi: 10.1038/ jcbfm.2013.186.

5. Khan ZP, Ferguson CN, Jones RM. alpha-2 and imidazoline receptor agonists. Their pharmacology and therapeutic role. Anaesthesia. 1999 Feb;54(2):146-65. PMID: 10215710.

6. Maier C, Steinberg GK, Sun GH, Zhi GT, Maze M. Neuroprotection by the $\alpha 2-$ adrenoreceptor agonist dexmedetomidine in a focal model of cerebral ischemia. Anesthesiology. 1993 Aug;79(2):306-12. PMID: 8102042.

7. Kuhmonen J, Pokorný J, Miettinen R, Haapalinna A, Jolkkonen J, Riekkinen $P$ Sr, Sivenius J. Neuroprotective effects of dexmedetomidine in the gerbil hippocampus after transient global ischemia. Anesthesiology. 1997 Aug;87(2):371-7. PMID: 9286902.

8. Cosar M, Eser O, Fidan H, Sahin O, Buyukbas $S$, Ela $Y$, Yagmurca $M$, Ozen OA. The neuroprotective effect of dexmedetomidine in the hippocampus of rabbits after subarachnoid hemorrhage. Surg Neurol. 2009 Jan;71(1):54-9; discussion 59. doi: 10.1016/j.surneu.2007.08.020.

9. Huang $R$, Chen $Y, Y u$ AC, Hertz L. DEXinduced stimulatio $\mathrm{n}$ of glutamine oxidation in astrocytes: a possible mechanism for its neuroprotective activity. J Cereb Blood Flow Metab. 2000 Jun;20(6):895-8. PMID: 10894172.
10.Xie C, Wang Z, Tang J, Shi Z, He Z. The effect of DEX post-treatment on the inflammatory response of astrocyte induced by lipopolysaccharide. Cell Biochem Biophys. 2015 Jan;71(1):407-12. doi: 10.1007/ s12013-014-0213-0.

11.Longa EZ, Weinstein PR, Carlson S, Cummins R. Reversible middle cerebral artery occlusion without craniectomy in rats. Stroke. 1989 Jan;20(1):84-91. PMID: 2643202.

12.Shang Y, Gu PF, Shang Y, Li Y. Penehyclidine hydrochloride inhibits glutamate release and related research in global brain ischemia/ reperfusion rats. Zhongguo Ying Yong Sheng Li Xue Za Zhi. 2011 Aug;27(3):353-6. PMID: 22097734.

13. Rees S, Harding R, Walker D. The biological basis of injury and neuroprotection in the fetal and neonatal brain. Int J Dev Neurosci. 2011 Oct;29(6):551-63. doi: 10.1016/j. ijdevneu.2011.04.004.

14. Mao X, Ji C, Sun C, Cao D, Ma P, Ji Z, Cao F, Min D, Li S, Cai J, Cao Y. Topiramate attenuates cerebral ischemia/reperfusion injury in gerbils via activating GABAergic signaling and inhibiting astrogliosis. Neurochem Int. 2012 Jan;60(1):39-46. doi: 10.1016/j. neuint.2011.10.015.

15. Takeuchi H, Jin S, Suzuki H, Doi Y, Liang J, Kawanokuchi J, Mizuno T, Sawada M, Suzumura A. Blockade ofmicroglial glutamate release protects against ischemic brain injury. Exp Neurol. 2008 Nov;214(1):144-6. doi: 10.1016/j.expneurol.2008.08.001.

16. Kimelberg HK, Nestor NB, Feustel PJ. Inhibition of release of taurine and excitatory amino acids in ischemia and neuroprotection. Neurochem Res. 2004 Jan;29(1):267-74. PMID: 14992286.

17. Kostandy BB. The role of glutamate in neuronal ischemic injury: the role of spark in fire. Neurol Sci. 2012 Apr;33(2):223-37. doi: 10.1007/s10072-011-0828-5.

18.Brouns R, De Deyn PP. The complexity of neurobiological processes in acute ischemic stroke. Clin Neurol Neurosurg. 2009 Jul;111(6):483-95. doi: 10.1016/j. clineuro.2009.04.001. 
19.Doyle KP, Simon RP, Stenzel-Poore MP. Mechanisms of ischemic brain damage. Neuropharmacology. 2008 Sep;55(3):310-8. doi: 10.1016/j.neuropharm.2008.01.005.

20.Gao J, Wang H, Liu Y, Li YY, Chen C, Liu LM, Wu YM, Li S, Yang C. Glutamate and GABA imbalance promotes neuronal apoptosis in hippocampus after stress. Med Sci Monit. 2014 Mar 27;20:499-512. doi: 10.12659/ MSM.890589.

21.Gao Q, Ji ZH, Yang $Y$, Cheng $R, Y u$ XY. Neuroprotective effect of Rhizoma Atractylodis macrocephalae against excitotoxicity-induced apoptosis in cultured cerebral cortical neurons. Phytother Res. 2012 Apr;26(4):557-61. doi: 10.1002/ ptr.3595.

22. Hasan N, McColgan P, Bentley P, Edwards RJ, Sharma P. Towards the identification of blood biomarkers for acute stroke in humans: a comprehensive systematic review. Br J Clin Pharmacol. 2012 Aug;74(2):230-40. doi: 10.1111/j.1365-2125.2012.04212.x.

23. Bekker A, Sturaitis MK. Dexmedetomidine for neurological surgery. Neurosurgery. 2005 Jul;57(1 Suppl):1-10; discussion 1-10. PMID: 15987564.

24.Tanskanen PE, Kyttä JV, Randell TT, Aantaa RE. Dexmedetomidine as an anaesthetic adjuvant in patients undergoing intracranial tumor surgery: a double $\bullet$ blind, randomized and placebo-controlled study. Br J Anaesth. 2006 Nov;97(5):658-65. PMID: 16914460.

25.Slosberg PS. Re: The neuroprotective effect of DEX in the hippocampus of rabbits after subarachnoid hemorrhage. Surg Neurol. 2009 Dec;72(6):648-9. doi: 10.1016/j. surneu.2009.02.002.

\section{Correspondence:}

Guangli Zhou

Department of Anesthesiology, Yantaishan

Hospital

Yantai 264001, China

Phone: +865356602199

Fax: +865356602199

guanglizhoudoc@163.com

Received: Feb 16, 2017

Review: Apr 19, 2017

Accepted: May 18, 2017
Conflict of interest: none

Financial source: none 